\title{
Frontières
}

\section{Vampires et avatars du mort-vivant}

\section{Transgression et banalisation}

\section{Deerie Sariols Persson}

Volume 23, numéro 2, printemps 2011

Enquêtes sur le cadavre : 2. Fantastique

URI : https://id.erudit.org/iderudit/1007590ar

DOI : https://doi.org/10.7202/1007590ar

Aller au sommaire du numéro

Éditeur(s)

Université du Québec à Montréal

ISSN

1916-0976 (numérique)

Découvrir la revue

Citer cet article

Sariols Persson, D. (2011). Vampires et avatars du mort-vivant : transgression et banalisation. Frontières, 23(2), 48-52. https://doi.org/10.7202/1007590ar d'utilisation que vous pouvez consulter en ligne.

https://apropos.erudit.org/fr/usagers/politique-dutilisation/ 


\section{VAMPIRES ET AVATARS DU MORT-VIVANT Transgression et banalisation}

Deerie Sariols Persson, Ph.D., professeure au Département SACIM (Sciences, Arts, Communication, Information, Multimédia), Université Versailles Saint-Quentin-en-Yvelines (France).

Le vampire est le monstre le plus populaire de notre époque, en littérature, cinéma et arts visuels. Il est une expression de l'impuissance humaine devant le passage du temps et la dégénérescence des corps, malgré les immenses avancées de la médecine. Les vampires montrent une face fascinante du mal et de l'éternité, dans une dialectique entre l'amour et la mort. Ils reflètent également l'individualisme matérialiste de l'homme contemporain pris dans un rêve de survivance physique et d'éternelle insatisfaction consumériste où le narcissisme du sujet est l'obstacle même de la relation avec l'Autre. Ces figures ténébreuses témoignent de certains aspects de l'ébranlement de bien des certitudes religieuses et spirituelles, comme l'idée d'une non-vie damnée mais puissante, confrontée à la mort chrétienne, liée au bien et au mal et à ses conséquences. Depuis le vampire de Byron ou celui de Stoker, jusqu'aux romans d'Anne Rice, Stephenie Meyer, ou les multiples versions cinématographiques (on peut citer les plus connues, celles de Murnau, Herzog ou Elias Merhige), les variantes du mythe du vampire montrent l'évolution des mentalités et pointent vers sa banalisation.
Le grand succès du vampirisme comme thème littéraire démarre vers le XVIII ${ }^{\mathrm{e}}$ siècle. Les Lumières de la raison et de la science éteignent petit à petit l'éclat de la foi. Mais le mystère de la mort est difficilement acceptable dans ce nouveau contexte matérialiste et dépourvu d'au-delà. Les anciennes superstitions continuent de consoler les hommes: « La négation de la transcendance a en fait été remplacée par "la religion de l'humanité", par "l'utopie d'un avenir radieux" propre aux doctrines matérialistes » écrit Claude Lecouteaux, dans Histoires de vampires (2002, p. 138). À cette époque charnière, les histoires de vampires sont légion et remplacent les chasses aux sorcières. Des légendes de vampires transylvaniens, comme celles du comte Dracula et de la comtesse hongroise Erzebeth Bathory, dont les tortures sur les domestiques et les amants étaient légendaires, sont de puissantes images du mal et de la mort, de bons candidats pour remplacer le vieux Satan. C'est à partir de ces histoires, divulguées surtout à partir du XVIII $^{\mathrm{e}}$ siècle, que les vampires passent de l'état d'êtres évanescents, semblables à des fantômes, à celui d'êtres dotés de corps très ressemblants aux humains vivants, avec des particularités extraordinaires de force et des capacités de métamorphose.

\section{LE MORT-VIVANT: POUR DISTRAIRE ET SE LAISSER DISTRAIRE}

Dans la littérature et au cinéma, les genres fantastique, science-fiction et d'horreur recréent soit des avatars du mort-vivant en tant qu'immortalité ratée, découlant de superstitions et de vieilles légendes-comme les vampires, les momies ou les zombies -, ou bien, au contraire, des êtres à l'immortalité réussie, comme les robots, les androïdes, les clones... La mode est au gothique et le look d'outre-tombe se banalise et s'esthétise. Le macabre vend bien. Ce n'est pas une simple utilisation de clichés, mais un retour aux sources. Comme si notre vie lisse et bourrée d'émotions audiovisuelles avait maintenant besoin de récupérer une partie de l'esprit ancestral.

La figuration de la mort de nos jours est en relation avec la distraction et l'amusement. En Occident, le décès se produit en général à un âge avancé, grâce aux progrès de la médecine et aux meilleures conditions de vie dont jouit la majeure partie de la population. La mort peut dès lors être envisagée sous des traits exotiques, ou comme un danger lointain, excitant mais sans risque réel. Que ce soit par une figuration tragique, dramatique, érotique ou comique, la mort est perçue comme un élément palpitant. L'attirance pour le morbide est largement exploitée par le fantastique. Devenir des spectateurs de la fin d'autrui 
serait une manière d'exorciser ce que l'on nie, la possibilité de la destruction de soi. Faire de l'humour sur la mort réveillerait une exaltation enfantine devant la mort des autres. On frôle la catastrophe et on y gagne l'exaltation du survivant. Le fantastique propose aussi des hypothèses pour satisfaire notre curiosité de savoir ce qu'il y a de l'autre côté. C'est de cette façon que le macabre trouve en nous tous des adeptes cachés.

Le rapprochement entre l'amour, le mal et la mort est en rapport avec la nature fragile du corps. Sa fugacité a toujours été exprimée par le lyrisme de l'ubi sunt: ce qui est jeune et désirable aujourd'hui finira poussière, comme tout le reste. Les morsures des vampires provoquent la mort ou transforment la victime en mort-vivant. Le thème du vampirisme est lié à l'érotisme et à l'amour sensuel non génital. Les tragédies d'horreur de l'époque romantique possèdent un caractère morbide; la passion y est exaltée par un dénouement fatal. Le Romantisme noir découvrait les liens puissants entre la beauté et le mal, ce qui comprenait tous les éléments en relation avec la mort: sadisme, masochisme, tendances assassines, sexualité déviée... ingrédients indéniables des histoires de vampires. Michel Guimar, dans son essai Principes d'une esthétique de la mort, insiste sur le fait que «le caractère fatal de la Mort vient illustrer une révélation de la plénitude de la source de Vie qu'est l'amour» (Guimar, 1988, p. 20). Le macabre rehausse le frisson érotique en le rattachant au danger, une manière d'accentuer le plaisir en se rapprochant artificiellement de la destruction.

Tant la littérature d'horreur, la sciencefiction que la fantasy, les jeux de rôle, les jeux vidéo, etc., ont multiplié les possibilités des avatars du mort-vivant. Aux vampires s'ajoutent des démons exotiques, comme les «goules» (variantes de démons arabes mangeurs de cadavres), les «liches» (des sorciers qui se maintiennent en vie par leurs propres pouvoirs) ou les «orques» de Tolkien. Ces êtres se présentent parfois (comme dans les films de Tim Burton,

\section{LE TEMPS ET LA MORT}

Le temps est «la substance même de notre être» (Fabre, 1992, p. 18). L'impossibilité de le maîtriser crée chez l'homme une angoisse primordiale. Cette peur n'est plus confortée par des certitudes religieuses, ce qui engendre un pessimisme existentiel et une course anxieuse vers le contrôle de la vie par des moyens scientifiques. Le développement de la médecine, le culte du corps ou la course au clonage en sont seulement quelques exemples. Dans la littérature et le cinéma, l'imagination se déchaîne dans l'étalage des aberrations ou idéalisations de cette lutte pour la prolongation de la vie. Les vampires, le monstre de Frankenstein et ses avatars morts-vivants, clones, super-héros de films et de bandes dessinées, sont toutes des créatures différentes qui proposent une réponse à l'angoisse de la mortalité. Le vampire agit souvent comme allégorie: "Symbole de l'intrusion de la mort et de l'au-delà par des voies sournoises et brutales dans un univers qui les exclut, le vampire représente l'inquiétude qui naît d'une rupture de l'ordre, d'une fissure, d'un décalage, d'une contradiction » (Lecouteux, 2002, p. 12).

Dans sa quête de l'immortalité, l'homme contemporain hésite aujourd'hui entre un matérialisme à outrance et une recherche de transcendance. Les progrès qui permettent le prolongement de la jeunesse et de la vie semblent mener à une vie qui n'a que son propre but et qui s'est coupée des traditions spirituelles qui nous promettaient un audelà après la mort. Les pratiques de préparation à une bonne mort héritées des générations antérieures ont

les romans vampiriques d'Anne Rice et le phénomène Twilight, de Stephenie Meyer) non comme l'ennemi d'un groupe en danger, mais comme une communauté presque normalisée, avec ses lois et ses habitudes aussi banales que les nôtres. été remplacées par un effort inouï pour
maintenir la vie. On accepte pour cela de subir l'agressivité de pratiques médicales parfois inhumaines. On prend des risques et on accepte de se mettre en danger pour se sentir vraiment vivants. 


\section{LES VAMPIRES MODERNES ET L'AMOUR QUI TUE}

Le vampire est probablement le monstre anthropomorphe le plus en vogue aujourd'hui. Il est à l'image de l'homme moderne qui cherche dans ce matérialisme amoral des moyens magiques pour rester jeune, et il va en subir les conséquences:

[...] l'homme à demi-mort ou l'énigme du mort-vivant (dont nous verrons combien elle se justifie également à travers le démantèlement de l'individu par la société et la dislocation du moi) est le thème central de notre temps. [...] Le symbolisme du vampire est celui de la vie dans la mort. C'est donc l'inverse de la mort sans après. Le vampire est un maudit: il préfère le salut de son corps au salut de son âme (Kappler, 1980, p. 119)

La zone de vie, ou plutôt de non-vie, des vampires est différente et bien pire que la mort véritable: nulle paix éternelle ni salut de l'âme, mais plutôt la perte de celle-ci. C'est l'anti-nature, l'immortalité damnée. La vie physique après la mort, antinaturelle, est définitivement associée au mal. Ce n'est pas la science qui s'en mêle, mais une ancienne damnation ou un sortilège qui remontent à la nuit des temps et qui varient selon les récits. La transgression de l'évolution physique des corps est d'abord un châtiment. Les récits de vampires, jusqu'au début du $\mathrm{XX}^{\mathrm{e}}$ siècle, soulignent le caractère sacrilège de l'activité vampirique. Comme Melmoth ou le Juif Errant, le vampire traverse les âges assez péniblement, exerçant son pouvoir meurtrier en même temps qu'il est poursuivi par les hommes.

Le vampirisme, comme absorption d'un corps de la même espèce, a des connotations différentes. Il est associé à la "pénurie alimentaire ou survie, vengeance ou épreuve, amour ou haine, destruction ou réintégration» (Thomas, 1984, p. 129). Il s'agit avant tout de manger ou de boire pour vivre ou survivre, et de s'emparer d'une partie de l'autre pour lui ressembler. Chez les créatures de la nuit, cela fait partie d'une nécessité contre nature qui leur donne des forces et une longévité surhumaines.

Ce côté marginal est parfois en contradiction avec d'autres sens possibles qui témoignent de la richesse des représentations du vampire. Son alimentation et sa rage meurtrière, toujours cachées par les voiles de la nuit, en font une image idéale pour des métaphores sociales et politiques. Les vampires symbolisent parfois ceux qui tyrannisent et profitent des autres. Il peut s'agir des moines (selon Voltaire), des capitalistes (selon Karl Marx), d'Adolph Hitler (selon l'écrivain Hans W. Geissendörfer) ou des Juifs (d'après Hans Heinz Ewers) (Lecouteux, 2002, p. 9). L'action de sucer le sang, donc la vie des hommes, est une métaphore de la tyrannie qu'exercent les puissants, à une période déterminée, vus selon une idéologie particulière.

Un corps mortel devient immortel par inoculation du sang d'un autre vampire; c'est une maladie qui se propage. Bien que certains vampires aient un charme hors du commun, comme celui de Byron ou Carmilla, d'autres comme le Dracula de Stoker possèdent une sorte de laideur élégante et hypnotique. Le cinéma a développé cet aspect monstrueux, avec des images masculines comme Nosferatu ou M. le Maudit. Les honnêtes gens peuvent être contaminés malgré eux par ces monstres, comme la pauvre Lucy et la chanceuse Mina, du roman de Stoker, qui réussit à échapper à l'état de mortevivante. L'important, encore à la fin du $\mathrm{XIX}^{\mathrm{e}}$ siècle, était de sauver l'âme. Les protagonistes de Dracula et d'autres récits vampiriques luttent contre la source de ce mal, quitte à en mourir. À cette époque, la mort héroïque est préférable à la lâcheté, et surtout à la deuxième mort, celle des morts-vivants. C'est l'idée même de cette infra-vie qui est insoutenable. Mina, se sachant contaminée, dit:

Si la mort ou la crainte de la mort constituaient les seuls obstacles, je n'aurais nulle crainte-qui ne m'envierait de pouvoir mourir parmi des amis qui feraient tout pour moi?

Mais la mort n'est pas le seul obstacle. Je ne puis croire qu'en mourant à présent, quand l'espoir s'ouvre à nous et que nous avons à accomplir une meilleure tâche, j'accomplisse la volonté de Dieu. C'est pourquoi, pour ma part, je renonce à la certitude d'une paix éternelle et accepte de m'enfoncer dans la ténèbre où se dissimulent sans doute les éléments les plus sombres que le monde, le monde inférieur puisse receler.

(Stoker, 2004, p. 433)

Cette deuxième vie / mort à laquelle elle va finalement échapper est «encore plus noire» que le repos éternel. La véritable mort veut dire la paix de l'âme, la conclusion du processus naturel de la naissance et du vieillissement. Faire preuve d'abnégation pour le bien de ses amis et de toute l'humanité fait partie de l'idéal victorien, où l'antinature et la marginalité sont dénoncées.

Même dans le film de Murnau, Nosferatu, eine Symphonie des Grauens (1921), on trouve des relents de cette morale. L'histoire est similaire à celle de Dracula, avec quelques changements. L'acteur Max Schreck, aidé de l'esthétique expressionniste, joue le monstre presque muet et d'une maladresse mécanique inquiétante. À la fin du film, le comte Orlok, le vampire, espionne Ellen, la jeune épouse du protagoniste, Hutter. Elle envoie son mari chercher du secours parce qu'elle veut le protéger. Lorsqu'ils sont seuls tous les deux, Orlok mord la jeune femme, mais le chant du coq le surprend: elle l'a fait attendre suffisamment pour le mener à sa perte. Le soleil se lève et Orlok est foudroyé par la lumière. Encore vivante, Ellen appelle Hutter. Il arrive en courant seulement pour la voir mourir. Le dernier panneau nous explique cette fin tragique mais salutaire: «Au même instant, comme par miracle, les morts cessèrent et, dans les rayons victorieux du soleil matinal, on vit disparaître l'ombre de l'oiseau de la mort. »Le mal disparaît grâce au sacrifice de cette femme, et le soleil souligne le caractère sacré de son acte.

L'homme occidental contemporain est moins porté à se sacrifier pour un paradis auquel il ne croit plus. Le triomphe du bien n'est même plus une option crédible, et l'ancienne lutte entre le bien et le mal est présentée comme une dynamique nécessaire au déroulement du monde. Cette position penche parfois vers un pessimisme apocalyptique: certaines réinterprétations du mythe soulignent ce dernier tabou brisé: les bons ne gagnent pas, ils n'échappent pas à la contamination vampirique et ils deviendront malgré eux, l'arme ultime de la destruction de l'humanité. Le Nosferatu de Herzog va dans ce sens, à la fois pessimiste et transgresseur: non seulement Mina meurt, mais Harker est possédé par l'esprit du monstre et il continuera à répandre le mal. Le roman I Am Legend, de Richard Matheson (1954) retourne encore plus la situation et s'achève avec

LA VIE PHYSIQUE APRÈS LA MORT, ANTINATURELLE, EST DÉFINITIVEMENT ASSOCIÉE AU MAL. CE N'EST PAS LA SCIENCE QUI S'EN MÊLE, MAIS UNE ANCIENNE DAMNATION OU UN SORTILÈGE QUI REMONTENT À LA NUIT DES TEMPS ET QUI VARIENT SELON LES RÉCITS. 
les yeux affolés des habitants de la nouvelle société, face au monstre humain qui les a décimés. Le bal des vampires, de Roman Polanski (1967), se moque des fins millénaristes qui signent leur appartenance au $\mathrm{XX}^{\mathrm{e}}$ siècle. Après toutes les mésaventures d'Alfred et de son professeur, leur fuite avec la belle Sarah ne sera pas une délivrance. Sans que personne ne s'en aperçoive, la jeune femme a été infectée et elle ne tarde pas à se jeter sur le pauvre Alfred, sans doute aussi sur le professeur. Au contraire, c'est grâce à «ce couple vampirisé que le mal va se répandre partout », souligne le narrateur. Le mal ne peut pas être arrêté.

On peut aussi relier ce sujet à d'autres questions qui ne se posaient pas auparavant, notamment le problème des limites de l'art. À notre époque, avec l'omniprésence de l'audiovisuel, il est devenu important de choquer et de transgresser, tant pour attirer le public que pour assouvir les souhaits profonds $\mathrm{du}$ réalisateur. L'ombre $d u$ vampire (Merhige, 2000) s'interroge subtilement sur ce sujet. Ce film raconte le tournage en 1921 de Nosferatu, de Murnau, chef-d'œuvre de l'expressionnisme allemand et référence incontournable du cinéma de terreur. Murnau (incarné par l'acteur John Malkovitch) prépare son film sur Dracula, mais doit en changer le nom pour des problèmes de droit d'auteur-ce qui est d'ailleurs un fait réel. Le vampire s'appelle donc Comte Orloch, et le film se présente comme Nosferatu, une autre dénomination du comte, pour éviter d'utiliser le nom Dracula. L'équipe allemande prépare ses acteurs et les extérieurs; nous assistons à la réalisation de certaines scènes très connues du film de Murnau comme l'entretien entre le comte et le jeune Hutter. Le héros laisse tomber une photo de sa fiancée Hélène, ce qui réveille la lubricité du vampire. Dans L'ombre du vampire, le monstre est reproduit de manière étonnante: même visage blême, mêmes griffes, même expression de folie que chez Murnau. Sa gaucherie se situe entre l'horrifiant et le comique; son comportement est invariable, que la caméra tourne ou

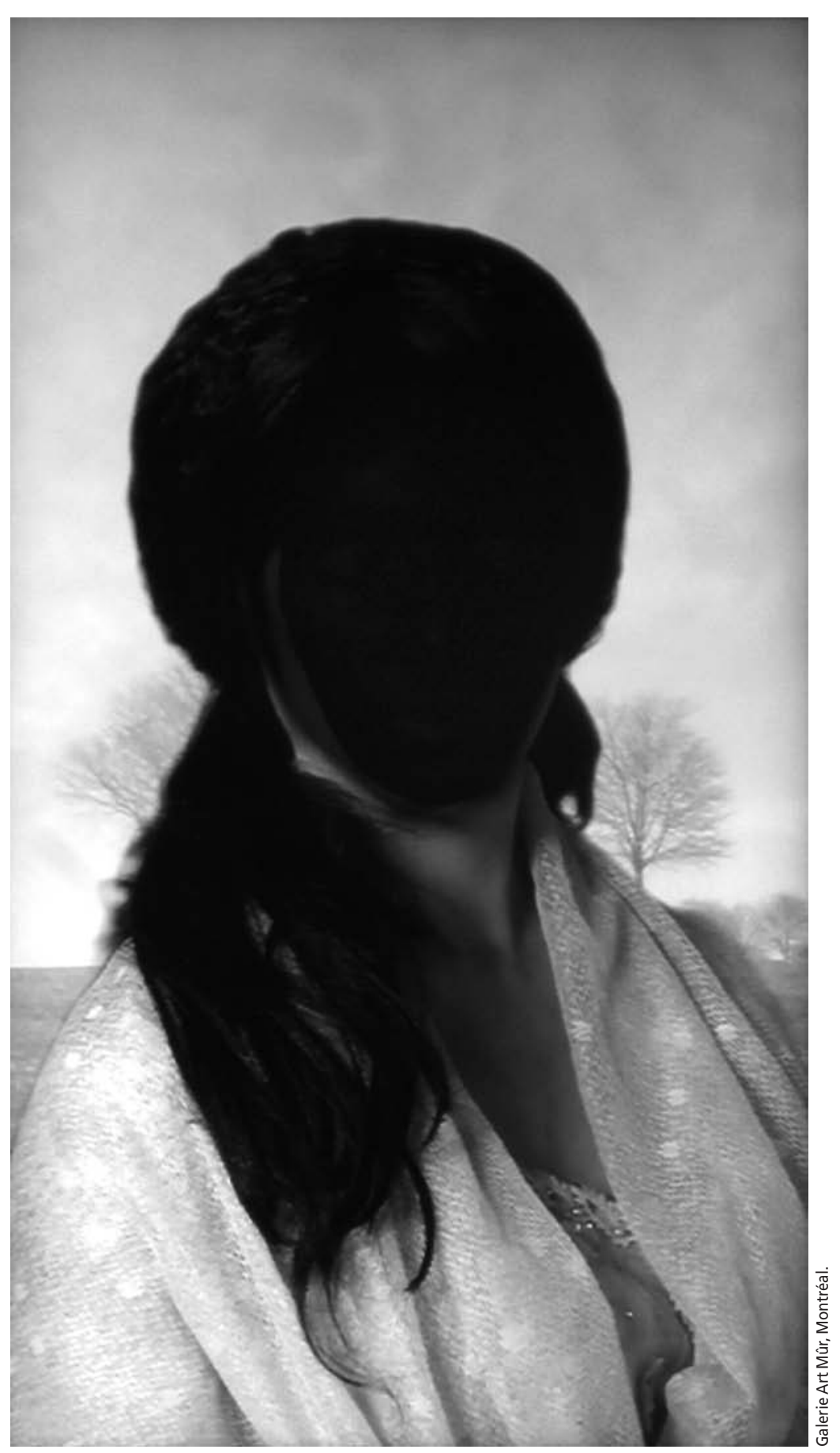

Nicholas et Sheila Pye, The Anti-Portraits, 2009, installation vidéo (partielle).

différentes versions pour pouvoir savourer cette nouvelle interprétation. Dans celle-ci, Murnau est un artiste maudit qui donnerait tout pour une œuvre parfaite: «Tout ce qui est en dehors de l'image n'a aucune existence», déclare-t-il. Pour lui, le cadrage de la caméra définit la réalité. Et effectivement, la réalité se mélange avec la fiction: il n'a pas engagé un acteur pour incarner Dracula, comme il le prétend. C'est un véritable vampire, à qui il a promis d'offrir la jeune protagoniste, en échange de sa prestation dans le film.

Dans L'ombre du vampire, Murnau crée un personnage féminin différent, plus érotique et pervers, plus ressemblant à la postérité du mythe: "Hélène est une femme qui découvre la plus haute expression de l'amour dans la plus exquise des souffrances imaginables », dit-il en donnant des conseils à l'actrice. Au lieu d'une douce et docile Ellen prête à se sacrifier pour son mari et pour l'humanité tout entière, comme dans Nosferatu, il en fait une sorte de femme sadienne, avide de nouvelles expériences. Le tournage finit mal: dans la scène de la mort d'Hélène et du comte, celui-ci mord véritablement l'actrice. Il survit aux balles qu'on tire sur lui devant la caméra fascinée de Murnau, qui tourne et tourne... jusqu'à la désintégration du vampire, à l'aube. Par ce dénouement, l'art est considéré comme plus important que la vie. L'image du corps en décomposition accélérée se mélange à celle de la pellicule qui brûle, usée par le geste excédé du réalisateur. Ses yeux sont ceux de la caméra, dont le regard absorbe l'image convoitée. C'est un regard avide mais mort, non humain. L'artiste devient un vampire de la

pas, ce qui éveille quelques inquiétudes dans l'équipe de tournage. L'approche est néanmoins différente, pour ne pas dire opposée entre les deux films. Dans la version d'Elias Merhige, on demande au spectateur de connaître le mythe sous réalité pour rendre celle-ci immortelle. Cette double mise en abyme montre la fascination par le croisement entre fiction et vérité et l'impossibilité pour l'artiste d'envisager une coupure entre les deux. Sa vie étant dédiée à l'art, il est devenu 
insensible et inhumain. L'art est ici l'équivalent de l'immortalité: c'est le film qui va rester pour toujours; les corps éphémères ne sont dignes que d'être ainsi immortalisés, les conséquences ne sont pas importantes. C'est un chef-d'œuvre qui est produit pour l'éternité, alors que les humains, vus comme de simples comparses de carton-pâte, peuvent être sacrifiés si cela permet de tourner une bonne scène. Cette variation du mythe l'enrichit en le plaçant dans l'ambivalence à plusieurs niveaux, éthique et esthétique, mais l'épuise à la fois en dévoilant les mécanismes de la création, son génie et ses carences morales.

Le vampire comporte sans doute aussi des caractéristiques qui en font un honnête reflet de l'homme contemporain. Son matérialisme foncier l'amène à lutter vigoureusement pour maintenir à tout prix son intégrité physique. Son immortalité n'est en fait qu'une longévité extraordinaire. La mort, vers laquelle il est attiré, est toujours au bout du chemin, soit par mégarde, soit par vengeance des hommes ou par volonté propre. Car le vampire postmoderne a mûri et il a réfléchi à son destin impossible. Il ne veut «se soumettre à personne, ni à Dieu, ni au diable et surtout pas à l'homme» (Rice, 1990, p. 121). D'un individualisme féroce, il évolue en solitaire ou en groupes où la hiérarchie maintient la rivalité. Pas d'enfants; le vampire se reproduit par morsure et ses rejetons sont presque toujours adultes (avec la fameuse exception de la petite Claudia, dans Entretien avec un vampire, d'Anne Rice [1990]). Si le vampire ancien était l'image de Satan ou du damné, ancré dans le silence, le moderne l'est souvent par choix et il en fait volontiers état dans son discours. Une manière de vendre son âme au diable comme on se lance vers le premier chirurgien esthétique venu, plein de promesses. Le vampire est le surhomme $\mathrm{du} \mathrm{XX}^{\mathrm{e}}$ siècle dont les avantages sont un leurre. Son impossibilité de créer son propre reflet dans un miroir fait référence à cette imposture.

Le vampirisme pourrait aussi se prêter à une analyse en tant que métaphore de l'amour impossible, celui où l'Autre devient soi-même. Les vampires sont des machines à désirer. Le thème du vampire moderne est très axé sur l'érotisme et sur l'insatisfaction. Le mode de vie occidental, très centré sur la consommation et l'envie incessante d'obtenir des objets, atteste d'un semblable inassouvissement.
Le vampire vit longtemps, mais seulement la nuit, avec les problèmes que cela comporte. Son alimentation cannibale doit être cachée aux hommes, pour éviter les persécutions. Le vampire est un paria, un marginal puissant mais puéril. Depuis le siècle dernier, l'image de cette créature de la nuit a rajeuni. Nosferatu et le comte de Stoker étaient presque des vieillards lubriques et d'une grande force physique. Les romans d'Anne Rice et de S.P. Somtow, par exemple, font des vampires de jeunes adultes au sommet de leur puissance sexuelle, dont le caractère possède encore des vestiges de l'adolescence. L'éternelle recherche de plaisir fait partie du quotidien du vampire et reste toujours inassouvie. Il a une presque-vie d'apparence et de frustration. Son processus de reproduction non génital coïncide avec son alimentation, sa source de force vitale et la clé de son immortalité. Il est un Dieu par sa proximité avec l'éternité et un animal, par ses mœurs sanguinaires.

Pour conclure, mentionnons que la publicité s'est elle aussi emparée du vampire. Dans un clip publicitaire diffusé il y a quelques années à la télévision, une bande de vampires se font bronzer au soleil avec des lunettes de soleil (d'une marque très connue) aussi branchées qu'eux. Un autre suceur de sang est marginalisé par le groupe parce qu'il ne possède pas de verres fumés. Dès que le soleil apparaît il se désintègre, ce qui déclenche l'hilarité de ses camarades. La normalité, c'est dorénavant d'appartenir au groupe qui suit la mode, quelle que soit sa particularité. La monstruosité ne fait plus problème; ce qui provoque le ridicule, c'est de ne pas savoir consommer les produits de la standardisation.

\section{Bibliographie}

FABRE, J. (1992). Le miroir de sorcière, Paris, José Corti.

GUIMAR, M. (1988), Principes d'une esthétique de la mort, Paris, José Corti.

KAPPLER, C.-C. (1980). Le monstre, pouvoirs de l'imposture, Paris, Presses universitaires de France.

LECOUTEUX, C. (2002). Histoire des vampires, Autopsie d'un mythe, Paris, Éditions Imago.

MATHESON, R. (2001 [1954]). Je suis une légende, trad. N. SERVAL, Paris, Denoël.

RICE, A. (1990). Lestat le vampire, Paris, Albin Michel.

RICE, A. (1990). Entretien avec un vampire, trad. T. MURAIL, Paris, JC Lattès.

STOKER, B. (2004). Dracula, trad. de J. FINNÉ, présentation et commentaires de C. AZIZA, Paris, Pocket.

THOMAS, L.-V. (1984). Fantasmes au quotidien, Paris, Klincksieck - Librairie des Méridiens.

\section{Filmographie}

HERZOG, W. (2010 [1979]). Nosferatu, fantôme de la nuit, DVD, couleur, Allemagne-France, Gaumont vidéo, 1 h 47.

MERHIGE, E. (2001 [2000]). L'ombre du vampire, DVD, couleur, É.-U.-France, Studio Canal, $1 \mathrm{~h} 29$.

MURNAU, F.W. (2007 [1921]). Nosferatu, DVD, N/B, muet sous-titré, Allemagne, Mk2, 1 h 35.

POLANSKI, R. (2004 [1967]). Le bal des vampires, DVD, couleur, É.-U., Warner Bros., $1 \mathrm{~h} 47$. 